\title{
Evaluation of measurement uncertainty of impact resistance of ceramic brick
}

\author{
Chen Weizhe ${ }^{1}$ \\ ${ }^{1}$ Quality and Technical Supervision and Inspection Center of Xuchang (National Quality Supervision and Inspection Center for \\ Ceramic Products of China (Henan)),Xuchang, Henan China
}

\begin{abstract}
As the economy continues to grow rapidly, people's requirements for product quality are increasing year by year, including ceramic tile products, which, as a typical building material product, are widely used in various large and small buildings. The quality of ceramic tile products is mainly evaluated through inspection and testing. As an important part of the test results, measurement uncertainty is an important parameter that characterizes the reliability of the measurement results. This article analyzes the sources of uncertainty in the measurement of ceramic tile impact resistance, uses the measurement results and related data, and draws the final conclusion.
\end{abstract}

\section{Introduction}

At present, China has developed into the world's largest manufacturer of building and sanitary ceramics. According to statistics, from January to December 2019, the main business income of the building ceramic industry above designated size was 307.99 billion yuan, and the national ceramic tile output was 10.161 billion square meters. Quantitative changes cause qualitative changes, and qualitative changes are inseparable from the improvement of detection technology, and measurement uncertainty is an important indicator that reflects the level of detection technology. JJF1059.1-2012 "Measuring Uncertainty and Expression" clearly stated that the final form of measurement results should be evaluated and expressed by uncertainty. The uncertainty of measurement results is becoming more and more common. Research on the evaluation of uncertainty of measurement results The results are endless, involving all aspects [1]-[3]. Therefore, this article takes the typical building material product - ceramic tile product as the research object, and carries out the evaluation and analysis of the uncertainty of ceramic tile impact resistance measurement.

\section{Measurement and analysis of impact resistance of ceramic tiles}

According to the GB/T 3810.5-2016 standard, the coefficient of restitution is used to determine the impact resistance of ceramic tiles.

\subsection{Measuring principle and equipment}

The measuring principle is to drop a steel ball from a fixed height on the sample and measure its rebound height to determine the coefficient of restitution. Measuring instruments and equipment include falling ball equipment, chrome steel balls and electronic timers.

\subsection{Measurement method}

\subsubsection{Concrete block}

The volume of the concrete block is about $75 \mathrm{~mm} \times 75 \mathrm{~mm} \times 50 \mathrm{~mm}$. Use this size mold to prepare the concrete block or cut it from a large concrete slab.

\subsubsection{Epoxy adhesive}

This adhesive should not contain toughening ingredients. A suitable adhesive is composed of 2 parts (by mass) of epoxy resin produced by the reaction of epichlorohydrin and diphenol propane and 1 part of activated amine as a hardener. The pure silica filler with an average particle size of 5.5 um measured by a particle counter or other similar methods is thoroughly mixed with other ingredients in an appropriate ratio to form a non-flowing mixture.

\subsubsection{Installation of test components}

Coat a layer of $2 \mathrm{~mm}$ thick epoxy resin adhesive evenly on the surface of the finished concrete block. Place three $1.5 \mathrm{~mm}$ diameter steel or plastic spacer marks in the 
middle of the three sides, so that each mark can be removed later. Press the specified sample face up onto the adhesive, and at the same time scrape off the excess adhesive before gently moving the three space marks. Before the test, place it for 3 days at a temperature of $23^{\circ} \mathrm{C} \pm 2^{\circ} \mathrm{C}$ and a humidity of $50 \% \mathrm{RH} \pm 5 \% \mathrm{RH}$. It can also be used for testing if the area of the tiles is less than $75 \mathrm{~mm} \times 75 \mathrm{~mm}$. Place a tile so that its center is consistent with the surface of the concrete, and then fill it with a tile to an area of $75 \mathrm{~mm} \times 75 \mathrm{~mm}$.

\subsubsection{Procedure}

Use the horizontal knob to adjust the falling ball equipment so that the steel frame is vertical. Place the test part under the electromagnet, and make the steel ball dropped from the electromagnet fall to the center of the test part which is fastened and positioned. Place a few eye components on the support, and place the front side of the sample horizontally. The steel ball falls from a height of $1 \mathrm{~m}$ and rebounds. The rebound height is measured by a suitable detection device and the recovery coefficient (e) is calculated. Another method is to let the steel ball jump back twice, record the time interval between the two rebounds (accurate to the millisecond level), calculate the rebound height, and then calculate the recovery coefficient.

Check whether there are any defects or cracks in the watch. All the slight cracks that cannot be observed with the naked eye or the eye wearing glasses at a distance of $1 \mathrm{~m}$ can be ignored. Note the bumps on the edges, but can be ignored when classifying tiles.

The above test steps should be repeated for the remaining test parts.

\subsubsection{Results presentation}

When a ball hits a stationary horizontal surface, its coefficient of restitution is calculated using equations (1)-(7):

$$
\begin{aligned}
& e=\frac{v}{u} \\
& \frac{m v^{2}}{2}=m g h_{2} \\
& v=\sqrt{2 g h_{2}} \\
& e=\sqrt{\frac{h_{2}}{h_{1}}} \\
& h_{2}=u_{0} t+\frac{g t^{2}}{2} \\
& t=\frac{T}{2} \\
& h_{2}=122.6 T^{2}
\end{aligned}
$$

$\mathrm{v}$ - The speed at the moment of departure (rebound), in centimeters per second $\mathrm{cm} / \mathrm{s}$ );

$\mathrm{u}$ - speed at the moment of contact, in centimeters per second $(\mathrm{cm} / \mathrm{s})$;

$\mathrm{h} 2$ - the height of the rebound, in centimeters $(\mathrm{cm})$;

$\mathrm{h} 1$ - The height of the falling ball, in centimeters (cm);

$\mathrm{g}$ - acceleration of gravity, $(=981 \mathrm{~cm} / \mathrm{s} 2)$; $(=0)$;

$\mathrm{u} 0$ - the speed when jumping back to the height point,

$\mathrm{T}$ - The time interval between two times, in seconds (s).

\section{Measurement uncertainty analysis}

\subsection{The main sources and analysis of uncertainty}

The main sources of uncertainty introduced in the measurement process are:

The random factors that affect the test results mainly include the variability of the measurement environment, the difference in personnel operations, the uncertainty of the falling ball equipment and test components.

\subsection{Mathematical model}

It is difficult to quantitatively study the influence of each influencing factor in actual evaluation. A relatively simple and easy method is to measure multiple samples of the same batch under repeatability measurement conditions, and calculate the standard deviation of the observation column as the composite repeatability uncertainty component of various random factors. Usually, the measurement result is multiplied by the repeatability factor frep, which is equal to 1 , and its standard deviation is equal to the relative combined standard uncertainty of the measurement result. Therefore, the mathematical model for evaluating uncertainty is shown in Equation 8:

$$
X=X_{i} \times f_{\text {rep }}
$$

\subsection{Evaluation of the uncertainty component introduced by measurement repeatability}

The measurement results are shown in Table 1. Table 1 Measurement results

\begin{tabular}{|c|c|c|c|c|c|c|c|c|c|c|}
\hline $\mathrm{N}$ & 1 & 2 & 3 & 4 & 5 & 6 & 7 & 8 & 9 & 10 \\
\hline $\mathrm{T}$ & & & & & & & & & & \\
$\mathrm{ms}$ & 792 & 784 & 769 & 793 & 802 & 807 & 778 & 784 & 786 & 799 \\
\hline & & & & & & & & & \\
\hline $\mathrm{h}_{2}$ & 76.90 & 75.36 & 72.50 & 77.10 & 78.86 & 79.84 & 74.21 & 75.36 & 75.74 & 78.27 \\
\hline $\mathrm{e}$ & 0.88 & 0.87 & 0.85 & 0.88 & 0.89 & 0.89 & 0.86 & 0.87 & 0.87 & 0.88 \\
\hline $\mathrm{A}$ & \multicolumn{70}{|c|}{0.87} \\
\hline
\end{tabular}

Where: 
The repeatability of the measurement is expressed by the standard deviation and calculated according to the Bessel formula:

$$
s(X)=\sqrt{\frac{\sum_{i=1}^{n}\left(X_{i}-\bar{X}\right)^{2}}{n-1}}=0.0133
$$

Since normally two samples are measured at a time, the average value is used to report the result. The standard deviation of the average value should be calculated as follows:

$$
s\left(\overline{X_{i}}\right)=u\left(\overline{X_{i}}\right)=\frac{0.0133}{\sqrt{2}}=0.0094
$$

The uncertainty of the measured repeatability factor frep is:

$$
u\left(f_{\text {rep }}\right)=\frac{s\left(\overline{X_{i}}\right)}{\overline{X_{i}}}=\frac{0.0094}{0.87}=0.01
$$

\subsection{Results report}

Because other uncertainty factors of this experiment are difficult to grasp quantitatively, the uncertainty of ceramic tile impact resistanceu $(x)=0.87 \times 0.01=0.01$.

\section{Conclusion}

Based on years of experience in the performance testing of ceramic tiles, the author of this article has carried out a detailed analysis of each uncertainty component that may appear in the process of measuring the impact resistance of ceramic tiles, and finally gives the final result through the calculation of the composite uncertainty. in conclusion.

The uncertainty evaluation method of ceramic tile impact resistance measurement proposed in this paper can be combined with actual application conditions to give a conservative uncertainty evaluation, which can be widely promoted and used.

\section{References}

1. Li Lingmei, Zhang Xin, Hu Jianhua, et al. Evaluation of measurement uncertainty with wooden straight (folding) ruler[J]. Foreign Electronic Measurement Technology, 2015, 34(4): 36-38.

2. Ling Mingyang, Li Huimin, Li Jiansheng, et al. Quasi Monte Carlo evaluation method of measurement uncertainty with correlation $[\mathrm{J}]$. Chinese Journal of Scientific Instrument, 2014, 5-13.

3. Ye Depei, Zhao Feng, Shi Changyan, etc., Measurement Uncertainty Evaluation and Table [5]. Beijing: China Metrology Press, 2012. 